\title{
Increased accuracy of signaling by hyperbolic odorant mixtures in a beneficial insect-plant relationship
}

Majid Ghaninia1, Anina C. Knauer², Florian P. Schiestl ${ }^{2}$, Tatyana O. Sharpee ${ }^{*}$, Brian H. Smith $^{1^{*}}$

1 School of Life Sciences, Arizona State University, 427 E. Tyler Mall, Tempe, AZ 85287, USA

2 Institute of Systematic and Evolutionary Botany University of Zurich, Zollikerstrasse 1078008 Zurich, Switzerland

3 The Salk Institute for Biological Studies, Computational Neurobiology Laboratory, North Torrey Pines Road, La Jolla, CA 92037, USA

${ }^{*}$ Co-equal senior and corresponding authors 


\section{Abstract}

Animals use odors in many natural contexts, for example, for finding mates or food, or signaling danger. Analyses of natural odors search for either the most meaningful components of a natural odor mixture, or they use linear metrics to analyze the mixture compositions. Both analyses assume that the odor space itself is Euclidian, like visual and auditory spaces. However, we have recently shown that the physical space for complex mixtures is 'hyperbolic' - curved - because of the correlations that arise in biosynthetic pathways. Here we shown that the shape of the space for flowers (Brassica rapa) using an existing data set can also be better described with a hyperbolic rather than a linear shape, and that components in the space correlate to the nectar and pollen resource sought by bee pollinators. We also show that honey bee and bumble bee antennae can detect most components of the $B$. rapa odor space. We argue that further investigation of the implications of hyperbolic space can have important implications for how sensory systems have evolved to encode the space. 


\section{Introduction}

Natural odors are typically mixtures of many different component ligands (Raguso, 2008). However, in spite of the high number of ligands in a natural odor, submixtures give rise to a low dimensional space that is hyperbolic (Zhou, Smith, \& Sharpee, 2018). The reason for this is that odorant concentrations in mixtures are not independent of each other, because subsets of them arise from the same or linked metabolic pathways within the plants. We argue that the low-dimensional and hyperbolic aspects of the space of natural odors could make it possible to deliver more reliable messages about the state of the plant important to pollinators than would be possible based on individual components, or on subsets analyzed with a Euclidean metric.

We focus here on floral odors produced by Brassica rapa, which is pollinated by honey bees (Apis mellifera) and bumble bees (Bombus sp). Floral perfumes of $B$. rapa contain as many as 26 different chemical constituents (Kobayashi et al., 2012). We show that bees can detect many or all of the odors in the mixture, and that several can contribute to perception of the mixture. However, the mixture gives a stronger, more reliable signal across a broader range of concentrations than the individual components. We further identified specific odorant submixtures that were correlated to the presence/absence of pollen and nectar. The submixtures computed within hyperbolic space had a greater predictive power about the concentration of pollen and nectar than those computed using a Euclidean metric. Our data are consistent with a model in which components of hyperbolic submixtures together amplify important, independent messages to bees about floral properties.

\section{Materials and methods}

\section{Experimental model and subject details}

In this study we used foragers of two bee species, honey bees (Apis mellifera carniolan) and bumble bees (Bombus terristris). The Apis mellifera forager bees were collected at the entrance of the hives located in the School of Life Sciences at Arizona State University, on Tempe campus, AZ, USA. The B. terristris forager bees, however, were captured from the entrance of the boxes of purchased colonies (Koppert Biological Systems; 
koppertus.com).

\section{Preparation and electroantennographic detection}

The collected foragers were immobilized by cooling and individually restrained in a row of eight truncated pipette tips ensuring that the heads and the antennae protruded from the narrow end of the pipettes. One antenna of the restrained bee was then cut off from the pedicel and subsequently transferred to a custom-made antennal holder composed of two capillary glass (tip and reference) electrodes (World Precision Instruments, Inc., USA) filled with insect ringer's solution. Each electrode was positioned alongside of the length of a microscope slide $(75 \times 25 \times 1 \mathrm{~mm})$ and secured on a small ball of dental wax. The tip and the cut base of the antenna were gently mounted between the tip and the reference electrodes, respectively (Supplementary Fig S1). The holder was then placed on the stage of an Olympus BX51WIF light microscope and viewed at $60 \times$ magnification for electroantennogram (EAG) experiments.

The EAG experiments were performed by inserting custom-made silver wires into the electrodes until the electrical recording with OSNs was attained. The EAG signal was amplified using an AC/DC differential amplifier (A-M Systems, Inc., Model 3000, USA), subsequently digitized using a digitizer (1440 A DigiData, Molecular Devices, USA), visualized and saved on a PC computer, and analyzed offline using Clampfit 10.3 software. The EAG responses were normalized (and presented in percent) to compensate for the plausible antennal fatigue caused by e.g. repeated delivery of various compounds and/or prolonged recording.

\section{Odorants}

Twelve selected compounds associated with Brassica rapa scent that were previously identified using chemical analyses (F.P. Schiestl, 2014; Taveira et al., 2009) were used in this study. Odors were 1-butene-4-isothiocyanate, $\alpha$-farnesene, methyl salicylate, indole, methyl benzoate, benzyl nitrile, (Z)-3-hexenyl acetate, p-anisaldehyde, phenylacetaldehyde, decanal, nonanal, acetophenone. Other characteristics of the compounds are displayed in Table 1. All compounds were diluted in hexane (Fisher Scientific, USA). 


\section{Odor delivery}

A $15 \mu$ l aliquot of the individual compounds was subsequently loaded onto two pieces of circular filter papers (10 mm diameter each, Whatman, UK) located in the tip of the Pasteur pipettes. The pipette tip was placed into a hole made along a delivery glass tube whose one end was hooked up to a stimulus controller (CS-55, Syntech, The Netherlands) through a Teflon tube and the other end was directed towards the preparation approximately $1-2 \mathrm{~cm}$ from it. The stimulus controller was set to deliver a $0.5 \mathrm{sec}$ of the individual stimuli into a constant $89 \mathrm{ml} / \mathrm{min}$ humidified and purified airstream passing over the preparation though the delivery glass tube. For every run, a new antennal preparation was used. To prevent the previously delivered odorant molecules from lingering in the antennal periphery, residual odors were fanned away for 30 sec after each odor delivery, and then another $30 \mathrm{sec}$ was allowed to pass ( 1 minute total time interval), before puffing the next compound. One experiment used a 5 minute interval.

\section{Dose response experiment}

All compounds were applied at increasing doses in linear steps ranging from $10^{1} \mathrm{pg} / \mathrm{l}$ to $10^{15} \mathrm{pg} / \mathrm{l}$. Different concentrations of each stimulus were tested only once (always from the lowest to highest concentrations) on individual antennae. Each concentration was tested at least three times and each time a new antennal preparation was used. For the complete mixture, $50 \mu \mathrm{l}$ of each of the twelve compounds at a given concentration were combined in a vial so that, at the end, the vial contained a total volume of $600 \mu l(50 \mu l$ of twelve compounds) at that concentration. Delivery of the compounds and analysis of the responses were done as described above. This dilution ensured that in the mixture, the same molar concentration of compounds was applied than for the individual compounds.

\section{Omission (subtraction) experiment}

For the omission experiment, we removed individual compounds from the $10^{13} \mathrm{pg} / \mathrm{L}$ complete mixture. The removal of a given compound in the mixture was compensated for by adding an additional $4.5 \mu \mathrm{l}$ of each of the 11 remaining compounds. In other words, to reach the total volume of $600 \mu \mathrm{l}, 54.5 \mu \mathrm{l}$ of each of the 11 remaining compounds (i.e. complete mixture minus compound $\mathrm{X}$ ) were combined in a separate vial. In total 13 
recordings from both species were made and for each recording a new antennal preparation/individual was used. After each recording, stimuli were randomized. Delivery of the compounds and analysis of the responses were done as described above.

\section{Floral odor sampling and analytical procedures}

Odor samples from B. rapa used herein were collected and first published in Knauer \& Schiestl (Knauer \& Schiestl, 2015), where a description of methods can be found.

\section{Statistics}

For the dose-response experiments we used data collected at $10^{13} \mathrm{pg} / \mathrm{L}$ as a reference point and applied both the Kruskal-Wallis test and ANOVA on both honey bees and bumble bees to compare the EAG response intensities for each of the Brassica odors. We used Tukey HSD post hoc test to confirm where the difference occurred between odorants. For the subtraction experiments we first applied both the Kruskal-Wallis test and ANOVA to compare the responses elicited by the incomplete mixtures (mixture with individual compounds omitted). In addition, we applied two sample t-tests to compare the responses elicited by the complete mixture (positive control) with those elicited by the incomplete mixtures. $P$ values lower than 0.05 were considered to be statistically significant. All results are represented as mean \pm SEM. The " $n$ " represents the number of antennae tested.

\section{Software}

Clampfit 10.3 was used to analyze the intensity of the EAG responses in $\mathrm{mV}$. The software may be available online at www.moleculardevices.com. We used R 3.3.0 for data entry and statistical analysis.

\section{Results}

The goal of this study was to compare flower-to-bee communication using either individual odorants produced by plants or their mixtures. Towards this goal, we first evaluated whether honey bees and bumble bees can detect a broad array of chemical constituents 
of $B$. rapa floral scent. The measurements were made based on electroantennogram (EAG) responses to each of the constituents and to a complete synthetic mixture (Ignell \& Hansson, 2004; Olsson \& Hansson, 2013). The EAG response to any odorant represents summed activity across all of the axons from thousands of Olfactory Sensory Neurons (OSNs) spaced along a bee's antenna (Supl Fig 1). Although EAG measurements are less sensitive than single sensillum recordings, they provide a more appropriate measure for determining whether OSNs on an antenna are collectively capable of detecting a large number of odor ligands, and EAG's provide a means to assess relative sensitivity to different odorants. The use of single sensillum recordings would be prohibitive for establishing a complete representation of odor ligands an antenna can detect, because of the large number of recordings that would be needed.

\section{Honey bees and bumble bees can detect most components of the mixture, which also gives the most robust response}

We tested each antenna from honey bees and bumble bees with an increasing concentration series of odor ligands diluted in hexane (Figs 1A and B; Supl Fig 2). We observed no response at concentrations up to $10^{11} \mathrm{pg} / \mathrm{L}$, at which point responses to some odors began to increase. By $10^{11}$ or $10^{13} \mathrm{pg} / \mathrm{L}$ both species' antennae responded to all odors, with the exception of 1-butene-4-isothiocyanate in honey bees. At higher concentrations responses to most pure ligands declined. This decline was likely due to sensory adaptation that persisted for at least the minute that lapsed between each stimulation. The sole exception was phenylacetaldehyde in honey bees, which showed no evidence of adaptation through $10^{15} \mathrm{pg} / \mathrm{L}$. We performed further EAG experiments on honey bees to determine how the timing between odor deliveries may have affected the responses to the highest $\left(10^{14}\right.$ and $\left.10^{15} \mathrm{pg} / \mathrm{L}\right)$ concentrations. We verified that when the OSNs are allowed enough time, i.e. five minutes, their responsiveness fails to show adaptation (Fig 1A).

Fig 1. Honey bees and bumble bees can detect almost all components of the Brassica mixture, but the mixture gives the most robust response. For the mixture, the $\mathrm{x}$-axis represents total molar concentration of solutes. Electroantennographic responses of $(A)$ honey bees (mean $\pm S E$ ) and $(B)$ bumble bees (mean $\pm S E, n=3$ ) to the 
scent of Brassica rapa. Note that the olfactory responses to most stimuli at $10^{14}$ and $10^{15}$ $\mathrm{pg} / \mathrm{L}$ are lower than those at $10^{13} \mathrm{pg} / \mathrm{L}$. The responses are restored after enough interdelivery time is given to the OSNs for them to be responsive again (right line-graphs in A). See Supl Fig 2 for statistical analysis.

It is worth noting that in both species the mixture elicited the most robust responses from $10^{13}$ through $10^{15} \mathrm{pg} / \mathrm{L}$. The concentration for the mixture refers to the sum of all 12 components. Thus the concentration of individual components in the mixture was only a fraction, approximately one-twelfth, of what was presented for each pure odorant at that concentration. There was little evidence of adaptation in honey bees to mixtures, even at the short interstimulus intervals. Adaptation was evident in bumble bees; nevertheless, even in this case, the mixture always elicited the highest response.

\section{Most components contribute to perception of the mixture}

To parse out the significance of specific components to the mixture we omitted the individual components from the mixture at $10^{13} \mathrm{pg} / \mathrm{L}$ (see Method Details). The logic was that the EAG response would decrease if a component contributes in any way - either qualitatively or quantitatively via intensity - to sensory processing of the mixture. Otherwise the response would be unchanged when the component is deleted.

We found that all components contribute to perception of the mixture. In both species, removal of one component significantly reduced the median normalized response to the mixture. The degree of reduction differed across compounds. For example, in honey bees (Fig 2A) the upper 75\% ranges after removal of alpha-farnesene, Z-3-hexenyl acetate, methyl salicylate and benzyl nitrile overlapped with the normalized response to the mixture, although the median values were still significantly below that level. In bumble bees (Fig 2B) the upper 75\% ranges for nonanal and 1-butene-4-isothiocyanate approached, but were still below, the normalized response to the mixture. The $75 \%$ ranges, and in many cases the maximum normalized responses, to all other mixtures with one component removed were below the full mixture response.

Fig 2. Several components contribute to perception of the mixture. Electroantennographic responses of honey bee (mean $\pm S E, n=11$ ) and bumble bee antennae (mean $\pm S E, n=6$ ) to the mixtures of Brassica rapa scent with individual 
component compounds omitted. Results show that in Apis mellifera omission of phenylacetaldehyde ( $t$-test, $t=2.23, \mathrm{df}=19, \mathrm{p}=0.03$ ), nonanal ( $t$-test, $\mathrm{t}=3.21, \mathrm{df}=17$, $\mathrm{p}=0.004$ ), decanal ( $t$-test, $\mathrm{t}=2.66, \mathrm{df}=19, \mathrm{p}=0.01)$, acetophenone $(t$-test, $\mathrm{t}=2.28, \mathrm{df}=19$, $p=0.03)$, and $p$-anisaldehyde ( $t$-test, $t=2.20, d f=19, p=0.03)$ from the blend significantly decreases the responses elicited by the OSNs (Fig 3A). Similarly, subtraction of the individual compounds including $\alpha$-farnesene $(t$-test, $t=2.53, \mathrm{df}=10, \mathrm{p}=0.02)$, acetophenone ( $t$-test, $\mathrm{t}=2.94, \mathrm{df}=8, \mathrm{p}=0.01)$, methyl salicylate ( $t$-test, $\mathrm{t}=2.26, \mathrm{df}=9, \mathrm{p}=0.04), \mathrm{p}$ anisaldehyde ( $t$-test, $t=2.37, \mathrm{df}=9, \mathrm{p}=0.04)$, and indole ( $t$-test, $\mathrm{t}=2.87, \mathrm{df}=6, \mathrm{p}=0.02)$, from the blend significantly impacts (reduces) the responsiveness of the OSNs in Bombus terrestris compared to the complete mixture (Fig 3B; ANOVA, $p>0.9$ ).

\section{Submixtures represent nectar and pollen content}

Since honey bees and bumble bees can perceive most if not all odors in the $B$. rapa mixture, we sought to establish the statistical relationships among the odors and how they correlate to important variables for bees, such as nectar and pollen. Different approaches have been used to perform this kind of comparison. Previous work by Knauer and Schiestl (Knauer \& Schiestl, 2015) identified two volatile compounds, phenylacetaldehyde and alpha-farnesene, that are positively associated with nectar and pollen, respectively. We used the same data set to investigate the possibility that submixtures may provide more complete information about nectar and pollen in $B$. rapa florets.

To find additional odor components that are associated with the nectar and pollen content of the flower, and which may supplement detection of those important resources for the bees, we performed two types of analyses. The first analysis was performed with standard Principle Component Analysis using the logarithms of odorant concentrations. This analysis identified odor combinations - i.e. submixtures - that account for largest variance across samples (Fig 3). The top three PC's cumulatively explained $80.6 \%$ of the total odor variance across the $B$. rapa florets and via factor loadings ( $\leq 0.01$ ) collectively accounted for all of the individual odorants. PC1 explained $52.1 \%$ of the variance and was positively associated with six odorants that increase or decrease together across florets: $\alpha$-farnesene (factor loading $=0.16)$, Z-3-hexenyl acetate (0.62), 1-butene-4isothiocyanate (0.21), methyl salicylate (0.50), methyl benzoate $(0.15)$, and panisaldehyde (0.48). PC2 explained $15 \%$ of the variance and was positively associated with phenylacetaldehyde (0.41) and indole (0.84). PC1 and PC2 were negatively associated with pollen-to-nectar ratio. Higher values on each PC were associated with 
more nectar than pollen. PC3 (13.2\%) described a positive association between nonanal (0.47), decanal (0.47), and acetophenone (0.35), but those three odorants together had a negative association with phenylacetaldehyde (-0.42). PC3 was positively correlated with pollen-to-nectar ratio. Higher values on PC3 corresponded to more pollen than nectar.

Fig 3. Pollen to nectar ratio. Both $P C 1(A)$ and $P C 2(B)$ showed a negative correlation with the ratio of log transformed pollen count to log transformed nectar volume, while PC 3 in the log transformed data set showed a positive correlation with this ratio $(\mathrm{C})$.

The PCA analysis assumes a Euclidian metric of the odor space (Koulakov, Kolterman, Enikolopov, \& Rinberg, 2011). We recently published a report that the odor space for different types of plant and animal derived volatiles is better fitted using a curved 'hyperbolic' metric (Zhou et al., 2018). The intuition for the relevance of the hyperbolic metric for natural odorants is that the hyperbolic metric approximates activity of hierarchical tree-like processes (Krioukov, Papadopoulos, Kitsak, Vahdat, \& Boguñá, 2010). Metabolic networks certainly have such characteristics. We therefore used hyperbolic Multi-Dimensional Scaling to embed points from B. rapa floral volatiles onto a 3D hyperbolic space. The curvature of the spaces was the same as in Zhou et al.(Zhou et al., 2018) with $\mathrm{R}_{\max }=7$ and $\mathrm{R}_{\min }=0.9$.

Embedding odorants into this hyperbolic space produced stronger correlations with nectar and pollen values compared to the analysis using PCA (Fig 4). In Fig 4A, points represent individual monomolecular odor components, and the distances between points reflect purely the correlation between these compounds across samples. Points that are more closely clustered tend to be more correlated across samples. In particular, two axes are strongly correlated with the pollen (blue) and log-nectar (red) content of the flower (Fig 4B,C). In Fig 4D, points represent individual flowers (i.e. measured odors mixtures). Here again one can identify two axes that are associated respectively with the log-nectar and pollen concentrations (Figs 4E,F). The correlations are stronger and more significant than those obtained in the Euclidean space using the PCA analysis.

Fig 4. Submixtures encode independent channels of information. (A) PC 1 in the logtransformed data set shows a significant positive association with pollen count per flower. PC 3 in the log transformed data set is negatively associated with nectar volume per flower $(B)$, and nectar sugars per flower (C). 


\section{Discussion}

Many odor mixtures serve as communication signals within and between species. In humans, the precise mixtures of the constituents are important for determining perceptual qualities of mixtures that relate to fruit pleasantness and food quality (Gilbert et al., 2015; Schwieterman et al., 2014; Zhou et al., 2018). At least in regard to the numbers of odorant receptors, many animals, including invertebrates, have olfactory systems that rival or exceed that of humans in complexity. The honey bee and fruit fly have 163 and 63 functional receptors in their genomes, resp. (Robertson \& Wanner, 2006; Robertson, Warr, \& Carlson, 2003). With the combinatorial nature of receptor responses to any odorant (Hallem, Ho, \& Carlson, 2004), which insects share with mammals (Nara, Saraiva, Ye, \& Buck, 2011), the perceptual space for odor coding is enormous (Gerkin \& Castro, 2015). Therefore, it is a reasonable hypothesis that many olfactory systems have evolved to analyze and parse different meanings out of complex natural odor mixtures. We propose that understanding the relationships between natural odors and the sensory systems that have evolved to detect them will require a statistical understanding of the structure of the odor landscape, much in the same way that similar analyses have been important for understanding vision (Bell \& Sejnowski, 1997; Ruderman \& Bialek, 1994) and audition(Lewicki, 2002).

In our original study (Zhou et al., 2018), we used published data from volatiles across several fruit varieties together with data from human psychophysical ratings for those fruit varieties (Gilbert et al., 2015; Schwieterman et al., 2014). Hyperbolic space provided better fits for both the volatiles and psychophysical preference data. The hyperbolic nature of the physical odor space has only been explored to date in relation to human perception. Here we use a beneficial insect-plant system related to pollination to test whether hypotheses that stem from this logic might be more general. $B$. rapa is pollinated naturally by honey bees, bumble bees and hover flies. When pollinated exclusively by bumble bees over several generations, the volatile profile of $B$. rapa is increased relative to when pollinated by hover flies (Gervasi \& Schiestl, 2017; F. P. Schiestl, Balmer, \& Gervasi, 2018). This pollinator-based change in odor profile shows the importance of odors in 
communication with insects that have well-developed olfactory systems, and it implies that there is a significant cost to odor production. Earlier analysis of the same dataset we used here identified a small subset of chemical components, especially phenylacetaldehyde and $\alpha$-farnesene , that were reliable and - because of the cost - honest signals of nectar and/or pollen (Knauer \& Schiestl, 2015).

The first requirement for this new hyperbolic framework for interpreting odor space is that most if not all components can be perceived by the sensory system and contribute to perception of the mixture. Our EAG analyses indeed show that most of the components of the mixture can be detected by the antennae of both honey bees and bumble bees. Interestingly, the mixture of all 12 components, each at a fraction of the concentration of the components, elicited the strongest response. Furthermore, at concentrations above $10^{13}$, antennae showed strong sensory adaptation to components. In honey bees the mixture did not elicit adaptation. In bumble bees the mixture elicited adaptation but responses were still the strongest at higher concentrations in spite of adaptation. These data provide one indication of why plants use mixtures of natural odors to attract bees. With a blend, more individual and different receptors are targeted, thus each individual receptor is exposed to lower doses of its cognate chemical compounds. Thus, less adaptation leads to more robust signaling over a larger range of concentrations.

The PCA and hyperbolic MDS analyses both point to combinations of odorants submixtures - that together can serve as particularly strong, independent indicators of the amount of nectar or pollen in a flower. Sometimes combinations are positive, indicating that observing two or more odorants together serve as a stronger signal than either alone. In other cases, the combinations are negative, meaning that the high levels of one or more odorants together with the absence or lower level of another odorant provides the strongest cue. These cue combinations could increase the reliability of honest signals of floral rewards (Knauer \& Schiestl, 2015). For example, due to non-linear competitive binding of different molecular components to odorant receptors, odor-evoked activity patterns are more stable and first-spike latencies are shorter for mixtures than for pure odorants (Chan et al., 2018). Both properties would enhance the accuracy and speed of detectability of mixtures over pure odorants. Competitive binding in mixtures can also predict more complex properties of sensory receptor responses, such as synergy, 
antagonism and overshadowing (Reddy, Zak, Vergassola, \& Murthy, 2018; Singh, Murphy, Balasubramanian, \& Mainland, 2019).

In summary, we have found that submixtures, which arise from covariance among the components from one odor object to the next, produce more robust sensory responses and are better predictors of floral resources than single components. While important information can be gleaned from evaluation of single components (Knauer \& Schiestl, 2015), our analyses reveal that there is far more information in odor mixtures that can be extracted by in early sensory processing. Improved predictions from the use of hyperbolic metric that we find here for $B$. rapa flowers indicates nonlinear synergistic interactions associated with the presence of multiple odorants. Put differently, in a hyperbolic space the presence of two components will exponentially increase the strength of the cue compared to the presence of individual components. Honey bees and bumble bees searching for pollen, nectar or both could focus on $B$. rapa odors that independently reflect levels of each reward. Our work also suggests further studies that would, for example, reveal the basis for correlations among components of submixtures - e.g. common biochemical pathways, genetic pleiotropy, epigenetic factors or linkage disequilibrium among genes that produce odor mixture components. It will also be fruitful to test the model using other coevolved odor/animal relations, such as pheromones, kairmones, aposematism, mimicry, or crypsis.

\section{Acknowledgements}

Dr. Kavita Sharma is acknowledged for her advice regarding EAG technique and Dr. Chris Jernigan for help with statistical analysis. We thank Kevin L. Haight for valuable comments on the first draft of the manuscript. This research was supported by Aileen Andrew Foundation, the National Science Foundation (NSF) award numbers IIS-1254123 and IOS-1556388 to TOS and 1556337 to BHS. Both awards from IOS resulted from an NSF Ideas Lab 'Cracking the Olfactory Code'. FPS and ACK were funded by the European Union's Seventh Framework Program ([FP7/2007-2013] [FP7/2007-2011]) under grant agreement no. 281093. 


\section{References}

Bell, A. J., \& Sejnowski, T. J. (1997). The "independent components" of natural scenes are edge filters. Vision Res, 37(23), 3327-3338.

Chan, H. K., Hersperger, F., Marachlian, E., Smith, B. H., Locatelli, F., Szyszka, P., \& Nowotny, T. (2018). Odorant mixtures elicit less variable and faster responses than pure odorants. PLoS Comput Biol, 14(12), e1006536. doi:10.1371/journal.pcbi.1006536

Gerkin, R. C., \& Castro, J. B. (2015). The number of olfactory stimuli that humans can discriminate is still unknown. Elife, 4. doi:10.7554/eLife.08127

Gervasi, D. D., \& Schiestl, F. P. (2017). Real-time divergent evolution in plants driven by pollinators. Nat Commun, 8, 14691. doi:10.1038/ncomms14691

Gilbert, J. L., Guthart, M. J., Gezan, S. A., Pisaroglo de Carvalho, M., Schwieterman, M. L., Colquhoun, T. A., ... O Olmstead, J. W. (2015). Identifying Breeding Priorities for Blueberry Flavor Using Biochemical, Sensory, and Genotype by Environment Analyses. PLoS One, 10(9), e0138494. doi:10.1371/journal.pone.0138494

Hallem, E. A., Ho, M. G., \& Carlson, J. R. (2004). The molecular basis of odor coding in the Drosophila antenna. Cell, 117(7), 965-979.

Ignell, R., \& Hansson, B. S. (2004). Insect olfactory neuroethology-an electrophysiological perspective Advances

in Insect Sensory Neuroscience (pp. 319-347).

Knauer, A. C., \& Schiestl, F. P. (2015). Bees use honest floral signals as indicators of reward when visiting flowers. Ecol Lett, 18(2), 135-143. doi:10.1111/ele.12386

Kobayashi, K., Arai, M., Tanaka, A., Matsuyama, S., Honda, H., \& Ohsawa, R. (2012). Variation in floral scent compounds recognized by honeybees in Brassicaceae crop species. Breed Sci, 62(4), 293-302. doi:10.1270/jsbbs.62.293

Koulakov, A. A., Kolterman, B. E., Enikolopov, A. G., \& Rinberg, D. (2011). In search of the structure of human olfactory space. Front Syst Neurosci, 5, 65.

doi:10.3389/fnsys.2011.00065

Krioukov, D., Papadopoulos, F., Kitsak, M., Vahdat, A., \& Boguñá, M. (2010). Hyperbolic geometry of complex networks. Phys Rev E Stat Nonlin Soft Matter Phys, 82(3 Pt 2), 036106. doi:10.1103/PhysRevE.82.036106

Lewicki, M. S. (2002). Efficient coding of natural sounds. Nat Neurosci, 5(4), 356-363. doi:10.1038/nn831

Nara, K., Saraiva, L. R., Ye, X., \& Buck, L. B. (2011). A large-scale analysis of odor coding in the olfactory epithelium. J Neurosci, 31(25), 9179-9191. doi:10.1523/JNEUROSCI.128211.2011

Olsson, S. B., \& Hansson, B. S. (2013). Electroantennogram and single sensillum recording in insect antennae Pheromone

Signaling (pp. 157-177): Springer.

Raguso, R. A. (2008). Wake up and smell the roses: the ecology and evolution of floral scent. Annual Review of Ecology, Evolution, and Systematics, 39, 549-569.

Reddy, G., Zak, J. D., Vergassola, M., \& Murthy, V. N. (2018). Antagonism in olfactory receptor neurons and its implications for the perception of odor mixtures. Elife, 7. doi:10.7554/eLife.34958 
Robertson, H. M., \& Wanner, K. W. (2006). The chemoreceptor superfamily in the honey bee, Apis mellifera: expansion of the odorant, but not gustatory, receptor family. Genome Res, 16(11), 1395-1403. doi:gr.5057506 [pii]

10.1101/gr.5057506

Robertson, H. M., Warr, C. G., \& Carlson, J. R. (2003). Molecular evolution of the insect chemoreceptor gene superfamily in Drosophila melanogaster. Proc Natl Acad Sci U S A, 100 Suppl 2, 14537-14542.

Ruderman, D. L., \& Bialek, W. (1994). Statistics of natural images: Scaling in the woods (Vol. 73, pp. 814). Physical Review Letters.

Schiestl, F. P. (2014). Correlation analyses between volatiles and glucosinolates show no evidence for chemical

defense signaling in Brassica rapa. (Vol. 2, pp. 10). Frontiers

in Ecology and Evolution.

Schiestl, F. P., Balmer, A., \& Gervasi, D. D. (2018). Real-time evolution supports a unique trajectory for generalized pollination. Evolution, 72(12), 2653-2668.

doi:10.1111/evo.13611

Schwieterman, M. L., Colquhoun, T. A., Jaworski, E. A., Bartoshuk, L. M., Gilbert, J. L., Tieman, D. M., . . Clark, D. G. (2014). Strawberry flavor: diverse chemical compositions, a seasonal influence, and effects on sensory perception. PLoS One, 9(2), e88446.

doi:10.1371/journal.pone.0088446

Singh, V., Murphy, N. R., Balasubramanian, V., \& Mainland, J. D. (2019). Competitive binding predicts nonlinear responses of olfactory receptors to complex mixtures. Proc Natl Acad Sci U S A, 116(19), 9598-9603. doi:10.1073/pnas.1813230116

Taveira, M., Fernandes, F., de Pinho, P. G., Andrade, P. B., Pereira, J. A., \& Valentão, P. (2009). Evolution of Brassica rapa var. rapa L. volatile composition by HS-SPME

and GC/IT-MS (Vol. 93, pp. 140-156). Microchemical Journal.

Zhou, Y., Smith, B. H., \& Sharpee, T. O. (2018). Hyperbolic geometry of the olfactory space. Sci $A d v, 4(8)$, eaaq1458. doi:10.1126/sciadv.aaq1458 


\section{TABLE 1: A comprehensive list of all materials and resources used to reproduce results presented in the manuscript.}

\begin{tabular}{|c|c|c|}
\hline REAGENT or RESOURCE & SOURCE & IDENTIFIER \\
\hline \multicolumn{3}{|c|}{ Chemicals, Peptides, and Recombinant Proteins } \\
\hline 1-Butene-4-isothiocyanate & Combi-Blocks & BATCH:A25781 \\
\hline a-Farnesene & ChemCruz & LOT:L0915 \\
\hline Methyl salicylate & SIGMA-ALDRICH & $\begin{array}{l}\text { LOT:MKBW9023 } \\
\mathrm{V}\end{array}$ \\
\hline Indole & $\begin{array}{l}\text { CHEM-IMPRX INT'L } \\
\text { INC }\end{array}$ & $\begin{array}{l}\text { LOT:001481- } \\
\text { 20120820 }\end{array}$ \\
\hline Methyl benzoate & Fluka & LOT:1339386 \\
\hline Benzyl nitrile & ALDRICH & LOT:BCBJ5504V \\
\hline (Z)-3-Hexenyl acetate & $\mathrm{TCl}$ & LOT:A5JXM-TA \\
\hline p-Anisaldehyde & $\mathrm{TCl}$ & LOT:R7QOK-TF \\
\hline Phenylacetaldehyde & $\begin{array}{l}\text { Santa Cruz } \\
\text { Biotechnology }\end{array}$ & LOT:A0616 \\
\hline Decanal & Alfa Aesar & LOT:101709990 \\
\hline Nonanal & Alfa Aesar & LOT:10179106 \\
\hline Acetophenone & Fluka & $\begin{array}{l}\text { LOT:BCBH8667 } \\
\text { V }\end{array}$ \\
\hline Hexane & Fisher Scientific & LOT:165552 \\
\hline \multicolumn{3}{|l|}{ Experimental Models: Organisms/Strains } \\
\hline Bumble bees (Bombus terristris) & $\begin{array}{l}\text { Koppert B.V., The } \\
\text { Netherlands }\end{array}$ & $\begin{array}{l}\text { www.koppert.co } \\
\text { m/pollination }\end{array}$ \\
\hline Honey bees (Apis mellifera carniolan) & $\begin{array}{l}\text { Maintained at Arizona } \\
\text { State University }\end{array}$ & www.asu.edu \\
\hline \multicolumn{3}{|l|}{ Software and Algorithms } \\
\hline Clampfit 10.3 & Molecular Devices, Inc. & $\begin{array}{l}\text { https://www.mole } \\
\text { culardevices.com } \\
\text { / }\end{array}$ \\
\hline $\mathrm{R}$ (Data analysis) & RStudio & $\begin{array}{l}\text { http://www.R- } \\
\text { project.org/ }\end{array}$ \\
\hline \multicolumn{3}{|l|}{ Other } \\
\hline Pipette tip & Fisherbrand & CAT:02-681-172 \\
\hline Borosilicate capillary glass & $\begin{array}{l}\text { World Precision } \\
\text { Instruments }\end{array}$ & LOT:1208336 \\
\hline Microscope slide & Fisherfinest & CAT:22-038-103 \\
\hline Dental wax & Surgident & LOT:1106012 \\
\hline
\end{tabular}


bioRxiv preprint doi: https://doi.org/10.1101/2020.05.13.093864; this version posted May 14,2020 . The copyright holder for this preprint (which was not certified by peer review) is the author/funder, who has granted bioRxiv a license to display the preprint in perpetuity. It is made available under aCC-BY 4.0 International license.

\begin{tabular}{|l|l|l|}
\hline BX51WIF light microscope & Olympus, Japan & SN:1H69084 \\
\hline AC/DC differential amplifier & $\begin{array}{l}\text { A-M Systems, Inc., } \\
\text { Model 3000, USA }\end{array}$ & SN:61676 \\
\hline Stimulus controller CS-55 & Syntech, Germany & $\begin{array}{l}\text { http://www.ocken } \\
\text { fels-syntech.com }\end{array}$ \\
\hline Digitizer & $\begin{array}{l}\text { 1440 A DigiData, } \\
\text { Molecular Devices, Inc., } \\
\text { USA }\end{array}$ & SN:814926 \\
\hline Filter paper & Whatman, UK & CAT:1004-070 \\
\hline
\end{tabular}




\section{Figure 2}

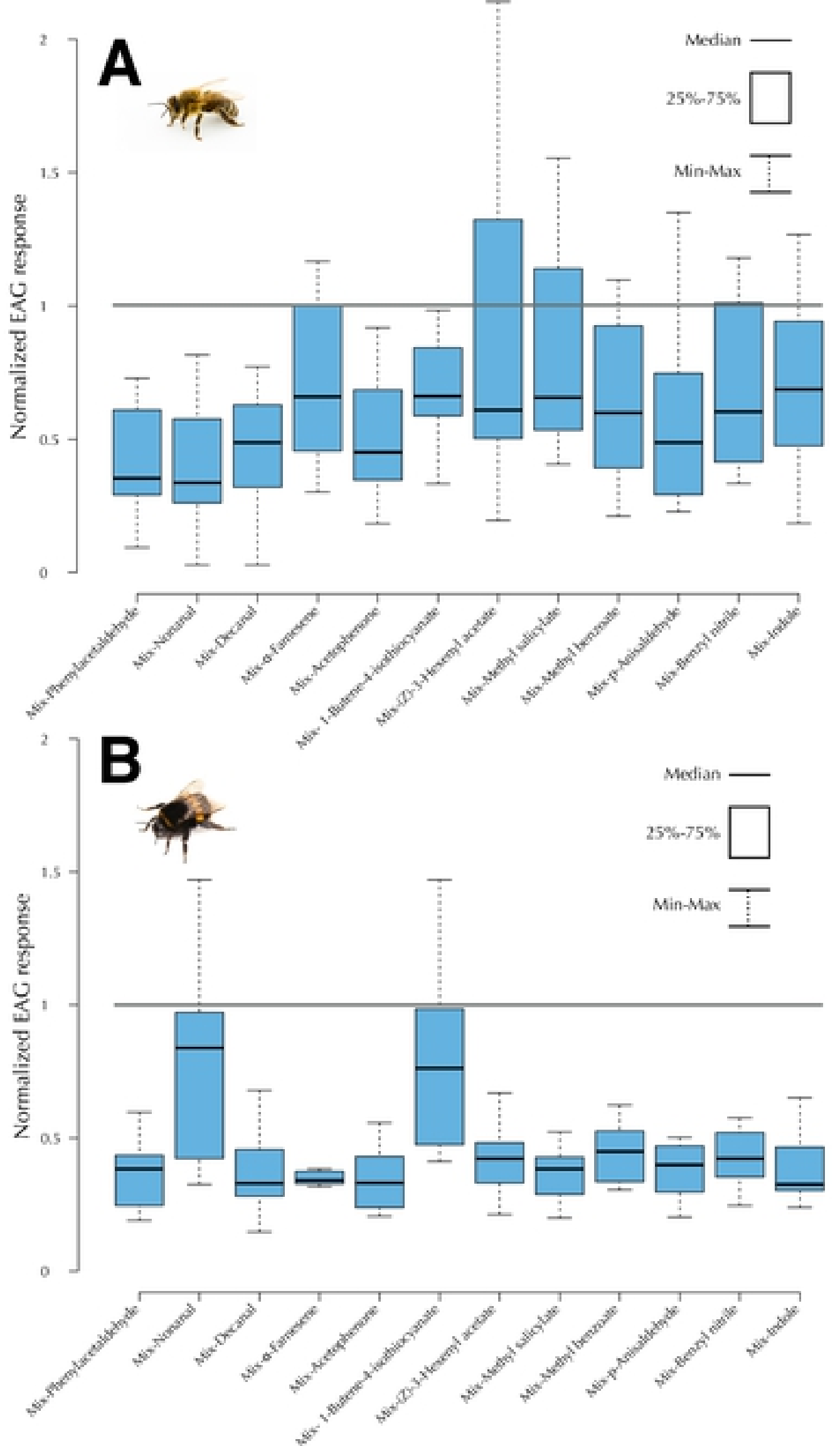




\section{Figure 3}
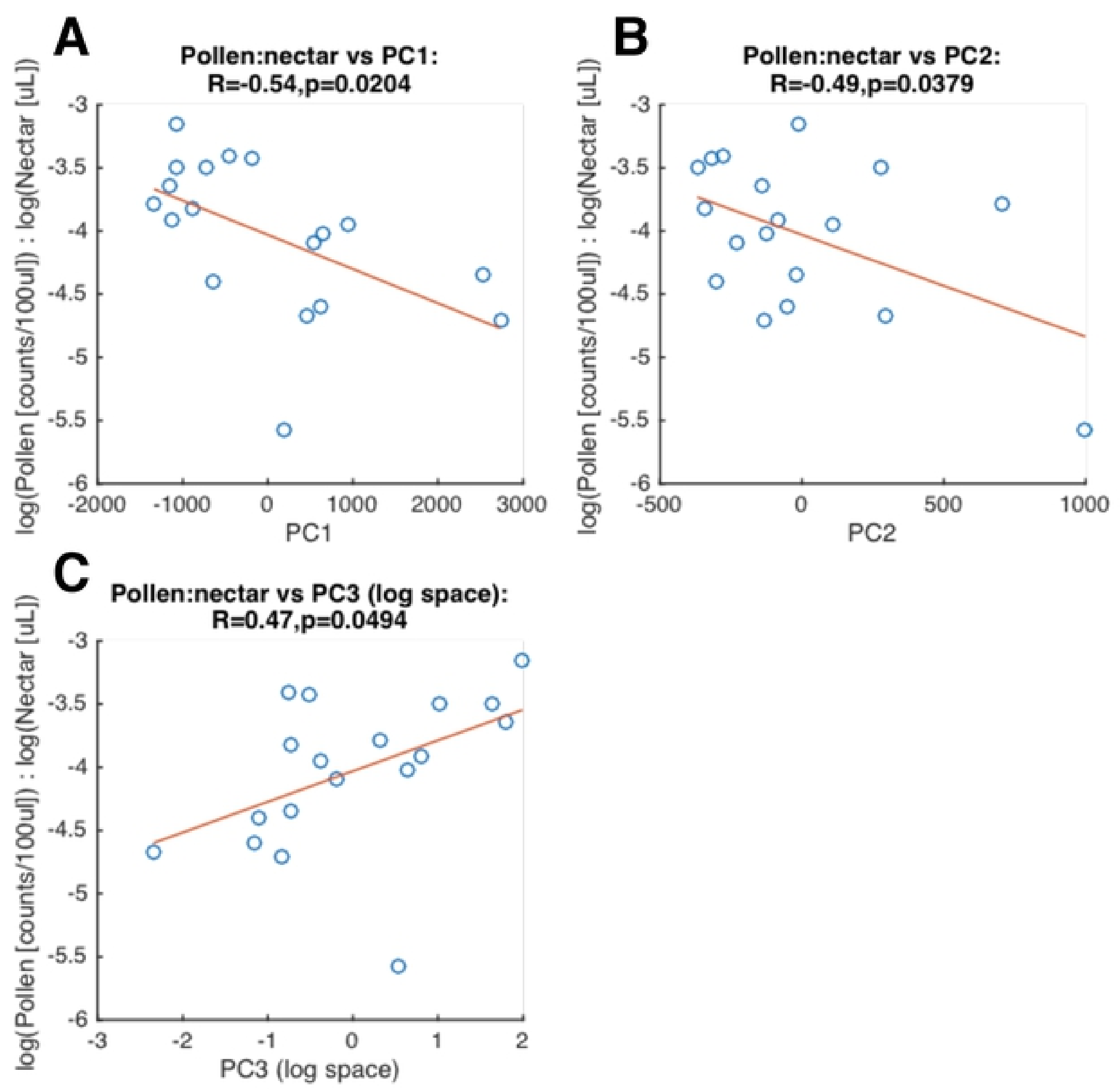

Fig 3 


\section{Figure 4}

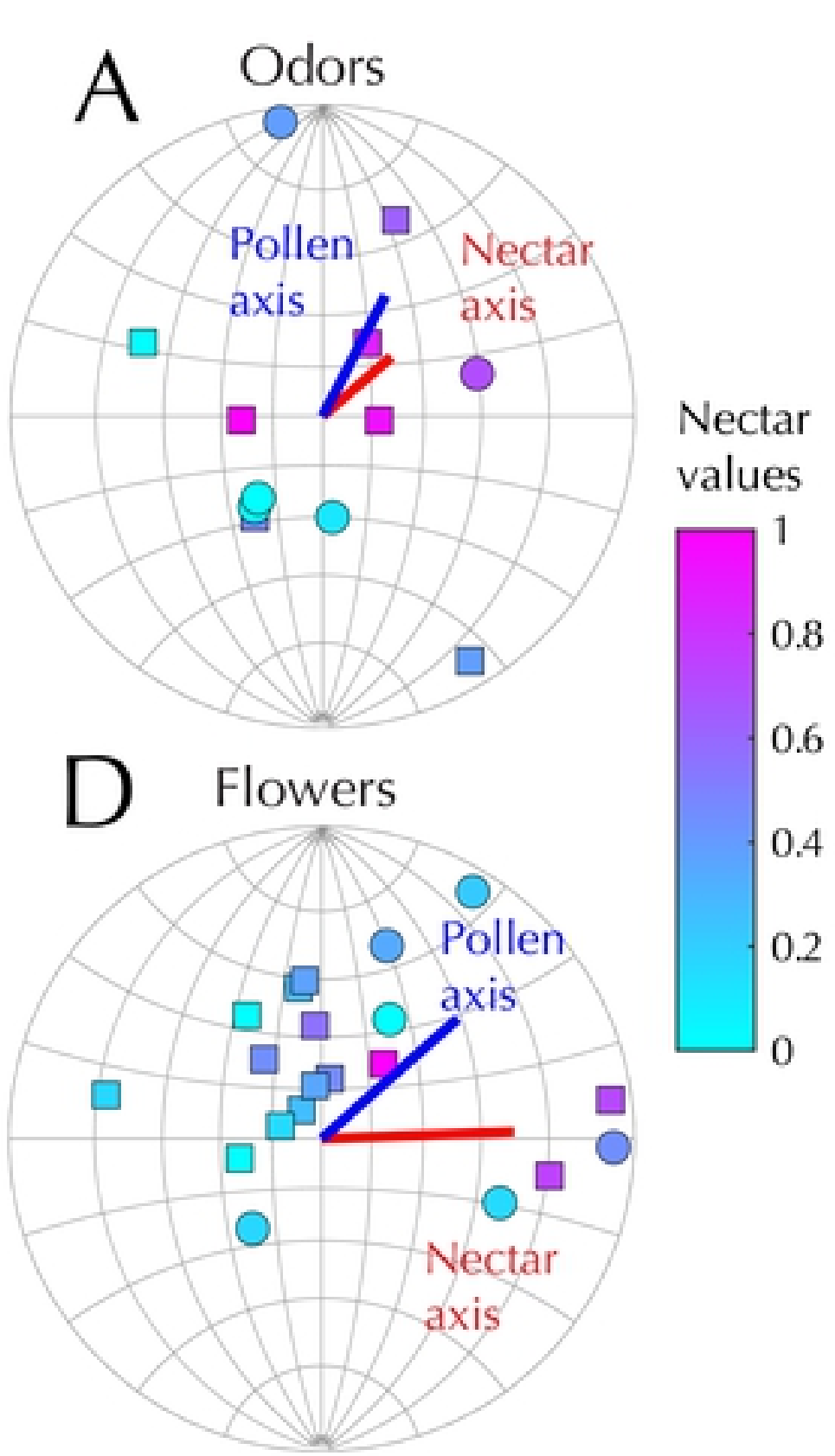

B
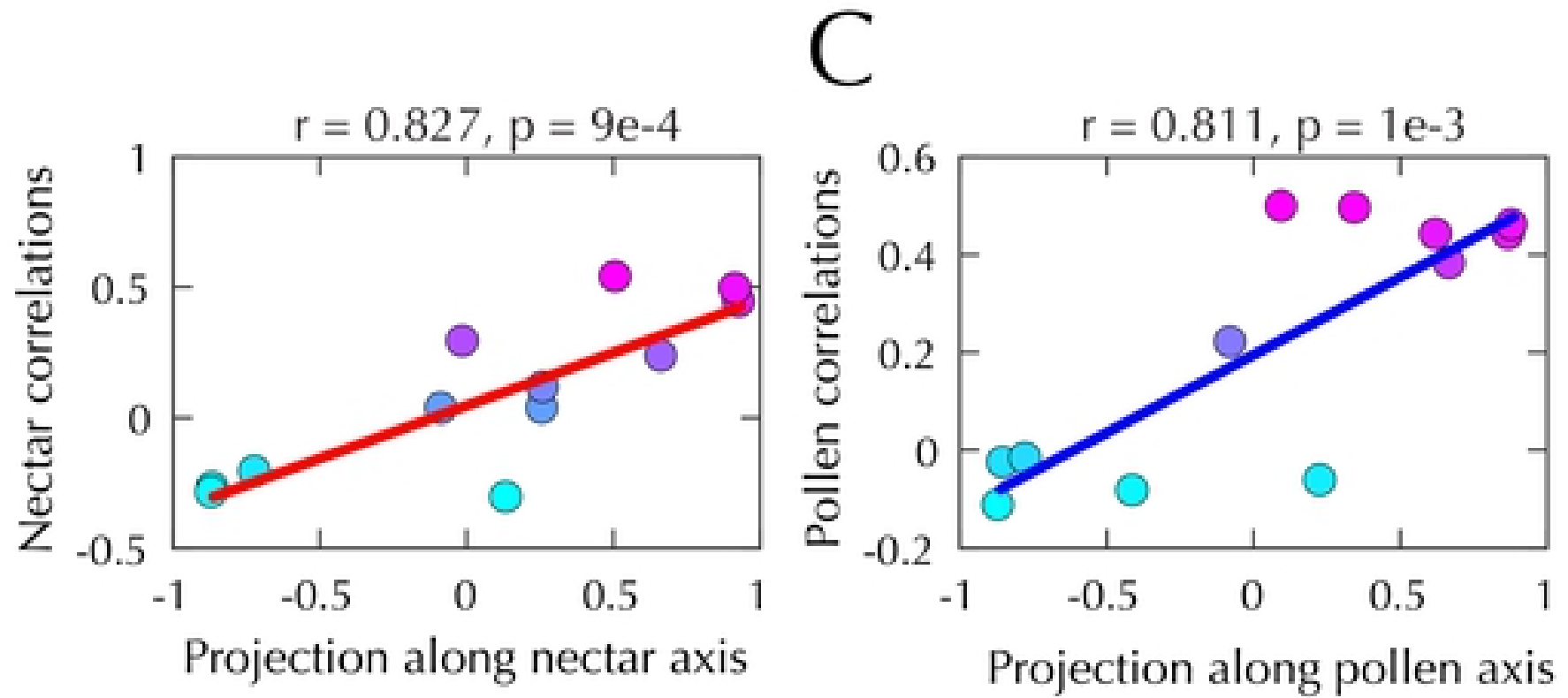

E
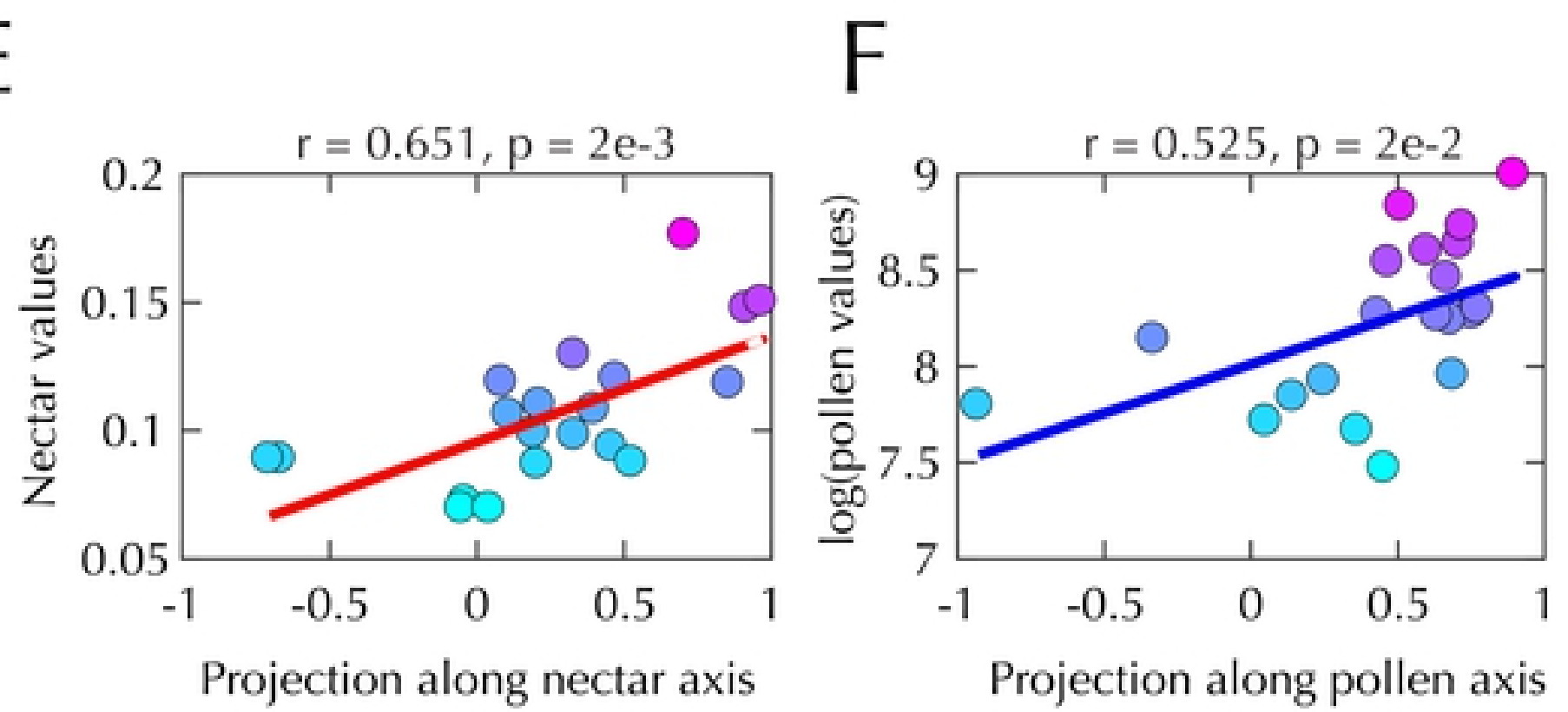

Fig 4 
